\title{
三谈氧化数
}

张颖, 张国艳, 权新军 ${ }^{*}$

吉林大学化学学院, 长春 130012

摘要: 首先对国内化学教材在描述氧化数概念时存在的不同说法进行讨论, 在此基础上建议用指定电荷数一词代替 形式电荷数(或形式荷电数)和表观电荷数来描述氧化数, 然后通过解读氧化数的定义, 得出若干重要推论, 最后介 绍了我们在编写无机化学教材时对氧化数部分内容所进行的探索与尝试, 并建议在无机化学、普通化学教材中引入 确定元素氧化数的价层电子数法。

关键词: 氧化数; 形式电荷数; 表观电荷数; 指定电荷数; 推论; 价层电子数

中图分类号: G64; O61

\section{The Third Discussion on the Oxidation Number}

Ying Zhang, Guoyan Zhang, Xinjun Quan *

College of Chemistry, Jilin University, Changchun 130012, China.

\begin{abstract}
In this paper, the different ideas about the concept of the oxidation number in domestic chemistry textbooks have been discussed. Based on this, we propose to use the term of specified charge number instead of formal charge number and apparent charge number to describe the oxidation number. Through the interpretation of the definition of the oxidation number, we draw some important inferences and finally introduce our explorations and attempts on the contents of the oxidation number in compiling the inorganic chemistry textbook, and suggest introducing the valence-shell electron number method to determine the oxidation number of elements in inorganic chemistry and general chemistry textbooks.
\end{abstract}

Key Words: Oxidation number; Formal charge number; Apparent charge number; Specified charge number; Inferences; Valence shell electron number

此前, 我们就氧化数的确定方法发表了两篇文章, 一篇针对与氧化数有关的教学中出现的歧义, 提出了自己的解决办法和确定元素氧化数的一项补充规则 ${ }^{[1]}$, 另一篇根据价层电子数与电子供需关 系, 提出了一种既无需对任何元素的氧化数进行指定、也无需考虑分子结构便能确定氧化数的新方 法 ${ }^{[2]}$ 。本文首先针对国内无机化学和普通化学教材在介绍氧化数概念时存在的不同表述进行分析, 提出我们的看法, 然后通过解读氧化数的定义, 得出若干重要推论, 最后谈一谈我们在编写《无机 化学简明教程》(第三版)时对氧化数部分进行的探索与尝试。

\section{1 关于氧化数概念的几种说法与我们的建议}

国内无机化学类和普通化学类教材对于氧化数定义的介绍或解释时存在以下几种情况:

(1) 将氧化数表述为化合物中元素的形式电荷(或形式荷电)数。 
（2）将氧化数表述为化合物中元素的表观电荷数。

(3) 上述两种情况以外的说法。

我们随机统计了 53 本 2000 年以来出版的教材, 单独采用形式电荷(或形式荷电)数说法的有 19 本, 占 $35.8 \%$, 单独采用表观电荷数说法的有 15 本, 占 $28.3 \%$, 同时采用形式电荷(或形式荷电)数 和表观电荷数两种说法的有 7 本, 占 $13.2 \%$, 其他情况有 12 本, 占 $22.6 \%$ 。严格地讲, 上面三种情 况是有区别的, 有必要澄清概念, 找到一个更准确的表达方式。

对于第一种情况, 我们认为, 无论是形式电荷数, 还是形式荷电数, 它们所表达的含义都是相 同的, 这部分编者的本意是说, 氧化数虽然是指化合物中元素或原子的电荷数, 但它并不一定是元 素或原子实际拥有的电荷数, 而只是一种人为规定的 “形式” 上的 “电荷数”。我们同意这样的看 法, 但认为据此就将氧化数表述为形式电荷数或形式荷电数的做法还是值得商榷的, 因为这里存在 着概念冲突。理由是, 形式电荷是化学中的一个专有名词, 而它与氧化数的意义是不同的, 新西兰 学者 John E. Packer 和 Sheila D. Woodgate 曾讨论过形式电荷与氧化数之间的区别 ${ }^{[3]}$ 。

所谓形式电荷, 是指分配给分子中一个原子的电荷, 并假定所有化学键中的电子在原子之间均 等共享, 而无论相对电负性如何 ${ }^{[4]}$ 。而任何化学实体中某元素的氧化数, 按照 IUPAC 的定义, 是指 如果该元素原子每个键中的电子被分配给负电性更高的原子, 则该元素的一个原子上将存在的电 荷 ${ }^{[5]}$ 。以 $C O$ 为例, 就形式电荷而言, $C$ 为 $-1, O$ 为 +1 ; 而就氧化数而言, $C$ 为 $+2, O$ 为 -2 。

对于第二种说法, 我们认为也不是十分恰当, 容易使人们产生误解。因为迄今为止, 表观电荷 还不是一个专有名词, 还没有明确的定义, 人们只能从字面上去理解它的含义。在汉语词典中, 表 观是指表面的, 或五官能感觉到的, 尤指视觉能感觉到的, 或以直接印象为基础的样子、性格或性 质 ${ }^{[6]}$ 。化学中已有一些含有 “表观” 的术语。例如, 强电解质在水中是完全解离的, 解离度本应为 $100 \%$, 但由于离子氛和离子对的存在, 实验测得强电解质溶液的解离度总是低于 $100 \%$, 显然, 实 验测出的解离度并不代表强电解质在溶液中的实际解离度, 故化学中将前者称作表观解离度; 再如,

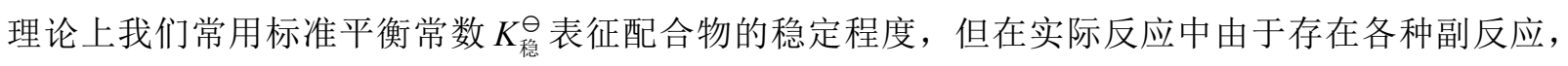

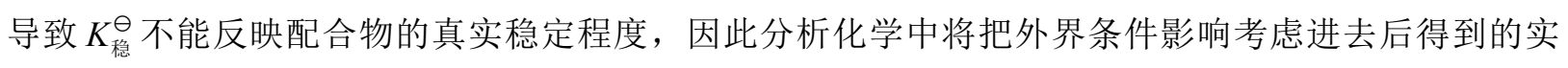
际稳定常数称为表观稳定常数。在这两个例子中, “表观” 已从原有的 “表面的、五官能感觉到的” 层面拓展到 “在一定条件下通过实验测定到的” 层面, 据此我们可以将表观电荷理解为能够被人们 察觉到或实验检测到的电荷。显然, 氧化数的真实含义与表观电荷数字面本身所表达的含义是有所 不同的。例如, 在 $\mathrm{CO}$ 中, 氧化数 $\mathrm{C}$ 为 $+2, \mathrm{O}$ 为 -2 , 并不意味着可以察觉或检测到 $\mathrm{C}$ 原子有 2 个正 电荷, $\mathrm{O}$ 原子有 2 个负电荷, 而是根据氧化数的定义人为地将 $\mathrm{C}$ 原子的 2 个电子分配给 $\mathrm{O}$ 原子后, $\mathrm{C}$ 原子就拥有了 2 个名义上的正电荷, 而 $\mathrm{O}$ 原子就拥有了 2 个名义上的负电荷, 尽管这种名义上的 电荷数与原子实际拥有的电荷数并不相同。

综上所述, 无论是用形式电荷 (或形式荷电) 数还是用表观电荷数来描述氧化数都是不恰当的, 从 IUPAC 给出的定义来看, 我们认为或许用 “指定电荷数” 这一新名词能更符合氧化数的含义, 因 为将元素原子每个键中的电子分配给负电性更高的原子, 其实就相当于指定了成键原子的电荷数。 当然, 这种 “指定” 并不是随意和没有根据的, 而是在服从电子供需关系的基础上, 与结构相统一 的电子分配。以 $\mathrm{HCOOH}$ 为例, 对于 $\mathrm{C}$ 原子而言, 共形成一个 $\mathrm{C}-\mathrm{H}$ 键, 一个 $\mathrm{C}-\mathrm{O}$ (差基氧)键和一 个 $\mathrm{C}=\mathrm{O}$ (端氧)键。根据电子供需关系, 在 $\mathrm{C}-\mathrm{H}$ 键中需要将 $\mathrm{H}$ 原子的 1 个电子分配给 $\mathrm{C}$ 原子, 在 $\mathrm{C}-\mathrm{O}$ 键中需要将 $\mathrm{C}$ 原子的 1 个电子分配给 $\mathrm{O}$ 原子, 在 $\mathrm{C}=\mathrm{O}$ 键中需要将 $\mathrm{C}$ 原子的 2 个电子分配给 $\mathrm{O}$ 原子, 净的结果就是 $\mathrm{C}$ 原子总共有 2 个电子被分配给其他原子, 相当于指定了 $\mathrm{C}$ 原子的电荷数为 +2 , 因此在 $\mathrm{HCOOH}$ 中 $\mathrm{C}$ 元素的氧化数为 +2 。 


\section{2 关于氧化数的若干重要推论}

根据 IUPAC 的氧化数定义, 我们可以得出以下推论:

(1) 同种元素原子之间成键对氧化数没有贡献。

(2) 在单质中, 元素的氧化数为零。

(3) 在中性分子中, 各元素氧化数的代数和等于零。

(4) 在单原子离子中, 元素的氧化数等于离子所带的电荷数; 在多原子离子中, 各元素氧化数的 代数和等于该离子所带电荷数。

对于推论(1), 其根据是同种元素原子之间由于电负性差值为 0 , 只能形成非极性共价键, 不允 许将成键电子硬性分配给任何一方, 只能平均分配, 其结果就是同种元素原子彼此之间成键对氧化 数没有贡献。例如 $\mathrm{H}_{2} \mathrm{O}_{2}$, 在考虑成键电子对氧化数的贡献时, 由于 $\mathrm{O}-\mathrm{O}$ 键中的 2 个电子只能每个 $\mathrm{O}$ 原子各自分配 1 个, 因而对氧化数没有贡献, 故只需考虑 $\mathrm{H}-\mathrm{O}$ 键对氧化数的贡献。

再看推论(2), 对于稀有气体单质, 由于它们以单原子分子形式存在, 元素的氧化数自然等于零; 对于其他元素的单质，推论(2)实际上是推论(1)的具体应用。

推论(3)和推论(4)则属于电荷守恒原理的必然结果。

上面四个推论是氧化数自身属性的反映, 对于元素氧化数的确定具有重要意义。

\section{3 在教材编写中对氧化数部分进行的探索与尝试}

在国内出版的《无机化学》《普通化学》类教材对于氧化数这部分内容通常只是介绍氧化数的 概念和确定氧化数的鲍林规则, 我们基于对氧化数性质和确定方法的研究体会, 在编写《无机化学 简明教程》(第 3 版)时尝试着对这部分内容进行了较大程度的改编。

我们是这样处理的: 首先介绍氧化数的概念, 然后介绍氧化数的若干重要推论, 最后介绍氧化 数的确定方法 ${ }^{[7]}$ 。之所以单独介绍氧化数的推论, 是为了帮助学生加深对氧化数性质的理解。尽管 推论(2)、(3)、(4)原本是氧化数确定规则的组成部分, 但是我们认为, 与其把它们看作是为了确定氧 化数而做的硬性规定, 倒不如把它们看作是氧化数自身的属性, 这样介绍反而更有利于对氧化数性 质的认识。

在介绍氧化数的确定方法时, 除了介绍鲍林的氧化数规则法外, 还介绍了我们提出的价层电子 数法。

对于前一种方法, 由于部分规则已经在氧化数推论中介绍, 在介绍氧化数规则法时不再重复。 另外, 我们还补充了一点, 即采用氧化数规则法确定氧化数时通常必须知晓化合物的结构, 否则遇 到比较复杂的化合物时可能会得出错误结果。若想在不知道分子结构的情况下准确判断包含过氧键 的化合物中元素的氧化数, 需要一条补充规则: 元素的正氧化数应小于或等于其价层电子的总数。

对于后一种方法, 由于是我们不久前刚刚提出的新方法, 这里简要地介绍一下。

由 IUPAC 的氧化数定义可以看出, 氧化数完全取决于成键两原子之间的电子供需关系。我们知 道, 只有价电子才能参与化学键的形成, 同时稀有气体具有稳定的电子结构, 这种稳定结构又总是 尽可能地体现在分子或离子中。因此, 元素的最高正氧化数受到其原子价层电子数的限制, 而元素 的最低负氧化数受到同周期稀有气体元素外层电子数与其价层电子数差值的限制, 据此提出用如下 方法确定元素氧化数。

对于二元化合物(分子或离子) $\mathrm{A}_{x} \mathrm{~B}_{y}^{n \pm}$ ( $n \pm$ 即 $n+$ 与 $n^{-}$, 分别表示正、负离子的电荷), 假设 $\mathrm{A}$ 的电 负性小于 $\mathrm{B}$ 的电负性, 若以 $a$ 表示 $\mathrm{A}$ 的价层电子数, $b$ 表示 $\mathrm{B}$ 达到稀有气体结构所需的电子数, 同 时考虑离子电荷的影响, 则有:

当 $x a \pm n=y b$ 时, $\mathrm{A}$ 的氧化数等于 $+a, \mathrm{~B}$ 的氧化数等于 $-b$;

当 $x a \pm n>y b$ 时, $\mathrm{B}$ 的氧化数等于 $-b$, 据此可求 $\mathrm{A}$ 的氧化数;

当 $x a \pm n<y b$ 时, $\mathrm{A}$ 的氧化数等于 $+a$, 据此可求 $\mathrm{B}$ 的氧化数。 
为了更清楚地说明问题, 这里举三个例子, 见表 1 。

表 1 二元化合物氧化数确定示例

\begin{tabular}{ccccccc}
\hline 化合物 & $a$ & $b$ & $\pm n$ & $\mathrm{~A}$ 氧化数 & $\mathrm{B}$ 氧化数 & 氧化数确定次序 \\
\hline $\mathrm{VO}_{2}^{+}$ & 5 & 2 & -1 & +5 & -2 & $\mathrm{~A} 、 \mathrm{~B}$ 同时确定 \\
$\mathrm{Fe}_{2} \mathrm{~S}_{3}$ & 8 & 2 & 0 & +3 & -2 & 先确定 $\mathrm{B}$, 后确定 $\mathrm{A}$ \\
$\mathrm{S}_{2} \mathrm{O}_{8}^{2-}$ & 6 & 2 & +2 & +6 & $-7 / 4$ & 先确定 $\mathrm{A}$, 后确定 $\mathrm{B}$ \\
\hline
\end{tabular}

对于三元和四元无机化合物以及小分子有机化合物, 也可以依据本方法确定元素氧化数。具体 步骤是: 对于能先拆分的化合物, 先拆分, 再进行判断。如 $\left(\mathrm{NH}_{4}\right)_{2} \mathrm{SO}_{4}$ 拆分成 $\mathrm{NH}_{4}^{+}$和 $\mathrm{SO}_{4}^{2-}$, 然后分 别按照二元化合物来判断。对于不能拆分的三元化合物 $\mathrm{A}_{x} \mathrm{~B}_{y} \mathrm{C}_{z}^{n \pm}$ (假设电负性 $\mathrm{A}<\mathrm{B}<\mathrm{C}$ ), 可先将 $x a \pm n$ 与 $z c$ ( $c$ 表示 $\mathrm{C}$ 达成稀有气体结构所需电子数)比较, 确定 $\mathrm{A}$ 或(和) $\mathrm{C}$ 的氧化数, 然后再确定其 余元素氧化数。例如 $\mathrm{SOCl}_{2}$, 电负性 $\mathrm{S}<\mathrm{Cl}<\mathrm{O}$, 其中 $\mathrm{S}$ 的价层电子数是 $6, \mathrm{O}$ 达到稀有气体结构所 需的电子数是 $2, a>c$, 可以先确定出 $\mathrm{O}$ 的氧化数为 -2 , 然后假设 $\mathrm{SOCl}_{2}$ 由 $\mathrm{O}^{2-}$ 和 $\mathrm{SCl}_{2}^{2+}$ 组合而成, 对 $\mathrm{SCl}_{2}^{2+}$ 按照二元化合物进行判断, 最终得出 $\mathrm{S}$ 的氧化数为 $+4, \mathrm{Cl}$ 的氧化数为 -1 。

将氧化数规则法与价层电子数法比较可以发现, 后者具有完全不需要考虑分子结构的优点, 不 管化合物的结构简单还是复杂, 都能对氧化数做出快速、准确的判断, 例外情况极少。这对于尚未 学过复杂化合物结构的大、中学生来说是十分方便、有效的。

\section{4 结语}

（1）建议在化学教学和教材中用指定电荷数一词代替形式电荷数、形式荷电数和表观电荷数来 描述氧化数。

(2) 由氧化数的定义可以得出若干重要推论, 这些推论是元素氧化数的自身属性, 在教学中单 独介绍有助于学生加深对氧化数性质的理解。

（3）价层电子数法确定氧化数具有完全不需要考虑分子结构的优点, 对于无机化合物以及小分 子有机化合物, 不管化合物的结构简单还是复杂, 都能对氧化数做出快速、准确的判断, 适合大、 中学化学教学, 建议将其引入化学教材之中。

\section{参 考 文 献}

[1] 张颖, 权新军. 大学化学, 2020,35(8), 103.

[2] 张国艳, 权新军. 化学通报, 2020, 83 (3), 277.

[3] Packer, J. E.; Woodgate, S. D. J. Chem. Educ. 1991, 68 (6), 456.

[4] 形式电荷, 百度百科. [ 2020-01-13]. https://baike.baidu.com/item/\%E5\%BD $\% \mathrm{~A} 2 \% \mathrm{E} 5 \% \mathrm{BC} \% 8 \mathrm{~F} \% \mathrm{E} 7 \% 94 \% \mathrm{~B} 5 \% \mathrm{E} 8 \% 8 \mathrm{D} \% \mathrm{~B} 7$

[5] International Union of Pure and Applied Chemistry. Commission on the Nomenclature of Inorganic Chemistry, 2nd ed.; Butterworth: London, 1971; pp. 5-6.

[6] 表观, 汉语词典-911 查询. [ 2020-03-07]. https://cidian.911cha.com/eWk5NA==.html

[7] 权新军, 张颖. 无机化学简明教程. 第 3 版. 北京: 科学出版社, 2020, in press. 